TECHNICAL NOTE

\author{
W.P. Daugherty \\ J.B. White \\ H.J. Cloft \\ D.F. Kallmes
}

\section{Rescue Retrieval of AngioGuard Embolic Capture System after Failure of Capture Sheath Retrieval in Carotid Angioplasty and Stenting}

\begin{abstract}
SUMmARY: This report describes the cases of 2 patients with failed retrieval of an AngioGuard distal protection device after carotid stent placement with rescue retrieval by use of a vertebral catheter. An AngioGuard was deployed before carotid angioplasty and stent placement. Attempted removal of the device with the provided recapture sheath was unsuccessful. A $5 \mathrm{~F} \mathrm{120-cm} \mathrm{vertebral} \mathrm{catheter} \mathrm{was}$ used to recapture and remove the AngioGuard. This technique is a simple and readily available solution for the retrieval of failed removal of a protection device.
\end{abstract}

S ymptomatic carotid stenosis is a disease process that has been traditionally treated with carotid endarterectomy and has been shown to be superior to medical management (the North American Symptomatic Carotid Endarterectomy Trial and the Asymptomatic Carotid Atherosclerosis Study). ${ }^{1,2}$ The first reported placement of a carotid stent was performed in the mid-1990s, ${ }^{3}$ but carotid stent placement has truly come into the realm of treatment of carotid artery disease with the advent of self-expanding stents and distal protection devices. In 2004, the SAPPHIRE trial was published, suggesting that carotid stent placement is at least equivalent to carotid endarterectomy in the measured outcomes of death, stroke, and myocardial infarction for the high-risk patient population enrolled in the study. ${ }^{4}$

Recently, the Precise RX microcatheter-based, self-expanding, nitinol stent (Cordis, Miami Lakes, Fla) received approval from the US Food and Drug Administration for use in carotid stenosis. With the application of this stent placement technology combined with balloon angioplasty and the AngioGuard (Cordis) distal protection device, this technique has allowed for the treatment of patients who are deemed at high surgical risk on the basis of their medical comorbidities. During the recent use at our institution of the AngioGuard distal protection device in 2 patients requiring carotid angioplasty and stent placement, the manufacturer-provided retrieval device failed to remove the protection device. In the cases of both of these patients, we were able to insert another sheath and remove the AngioGuard using a $5 \mathrm{~F}$ vertebral catheter without complication.

\section{Patients and Techniques}

We obtained institutional review board approval for this case series. Both of our patients were being treated for symptomatic carotid stenosis and were deemed relatively high risk for surgical treatment.

\section{Patient 1}

A right cervical carotid angioplasty and stent placement were being performed from a transfemoral approach. An AngioGuard $6 \mathrm{~mm}$ dis-

Received September 27, 2007; accepted after revision November 30.

From the Departments of Neurosurgery (W.P.D., J.B.W.) and Radiology (H.J.C., D.F.K.), Mayo Clinic Foundation, Rochester, Minn.

Please address correspondence to Wilson P. Daugherty, Department of Neurosurgery, Joseph 1-229, Mayo Clinic Foundation, Rochester, MN 55905; e-mail: daugherty. wilson@mayo.edu

DOI 10.3174/ajnr.A1118 tal protection device was guided through the stenosis and deployed. A $5 \times 20$-mm balloon was placed across the stenosis and was inflated to its nominal value. A Precise RX nitinol $8 \times 30-\mathrm{mm}$ stent was placed across the stenosis.

On trying to recapture the AngioGuard, we found that the capture device would not advance through the stent lumen. During each attempt, the distal portion of the capture sheath would bind in the lumen of the stent, preventing retrieval of the distal protection device. After several failed attempts to pass the retrieval device through the stent with both the AngioGuard and Accunet (Boston Scientific, Natick, Mass) retrieval sheaths, we decided to attempt retrieval using a $125-\mathrm{cm} 5 \mathrm{~F}$ vertebral diagnostic catheter passed over the $180-\mathrm{cm}$ AngioGuard wire. Because only approximately $60-\mathrm{cm}$ of the $180-\mathrm{cm}$ wire was exposed outside the guiding sheath, we were unable to pin or hold the wire as the diagnostic catheter was advanced. Even without pinning the wire, the AngioGuard basket and wire remained in stable position as the diagnostic catheter was advanced. Once the wire was exposed through the lumen of the diagnostic catheter, the wire was held in place as the catheter was advanced. The catheter was passed without difficulty through the stent and was advanced until it engaged the AngioGuard basket. The catheter and basket were removed without incident.

\section{Patient 2}

A left cervical carotid angioplasty and stent placement were being performed from a transfemoral approach. Because of tortuosity, we were unable to initially place the AngioGuard device. Therefore, we placed a buddy wire composed of a 0.035 -inch, stiff-angle Glidewire (Terumo, Tokyo, Japan) into the distal left common carotid artery, which allowed distal placement of the protection device. The buddy wire was removed and the protection device was deployed.

A $5 \times 20$-mm balloon was placed across the lesion and inflated to 6 atm for 30 seconds. The balloon was removed, and a Precise RX nitinol $8 \times 30$-mm stent was placed across the lesion. Several unsuccessful attempts were made to remove the protection device with the AngioGuard retrieval device. In this case, the retrieval device was able to be advanced beyond the stent; however, the device caught several times in the distal aspect of the stent at a prominent turn (Fig 1). The AngioGuard was redeployed and the retrieval device was removed. A $125-\mathrm{cm} 5 \mathrm{~F}$ vertebral catheter was advanced over the wire, and when access to the wire was again obtained, the catheter was advanced through the stent where the AngioGuard was recaptured without difficulty.

\section{Discussion}

With the increase in carotid stent placement for occlusive disease comes an increase in distal protection device deployment. 

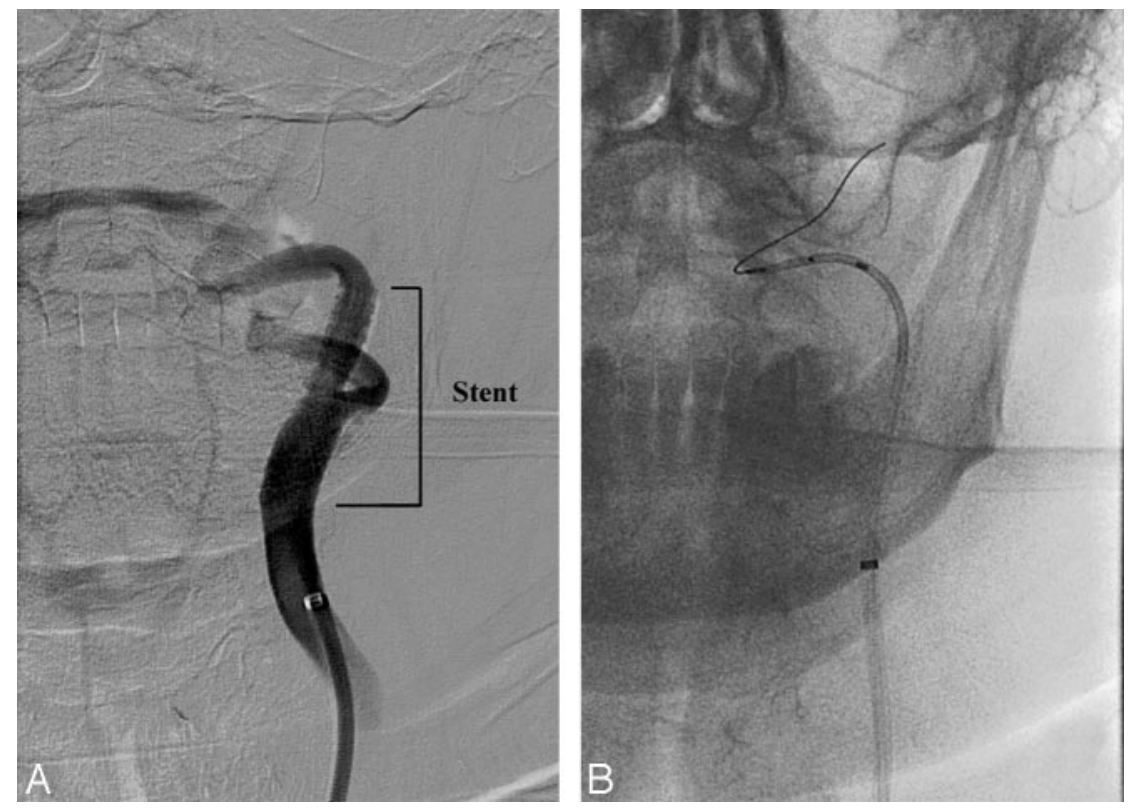

Fig 1. A, A common carotid artery digital subtraction angiographic examination after the deployment of the Precise RX stent with a prominent bend in the internal carotid artery distal to the stent. $B$, An unsubtracted image demonstrating the recaptured AngioGuard distal protection device within a $5 \mathrm{~F}$ diagnostic catheter.

Data from the SAPPHIRE trial ${ }^{5}$ suggest a $91.6 \%$ to $95.6 \%$ successful deployment and recapture of the AngioGuard distal protection device. These data were not further stratified to deployment versus retrieval difficulties. In the cases of our 2 patients, the difficulties arose from retrieval in both instances. In the first case, the retrieval device would not advance through the stent, which suggested a poststent placement irregularity of the artery or the conformation of the stent in the artery. The second case was complicated by an acute angle of the cervical internal carotid artery just distal to the cephalad aspect of the stent, causing the retrieval device to snag the stent on attempted removal, requiring redeployment of the AngioGuard. Dragging the distal protection device through the stent should be avoided to prevent possible fracture of the device or loss of previously captured debris that could embolize distally.

In the cases of both of our patients, we were able to advance a $125-\mathrm{cm} 5 \mathrm{~F}$ vertebral diagnostic angiography catheter over the $180-\mathrm{cm}, 0.014$ inch AngioGuard wire to retrieve the filter. This solution was easy and readily available and allowed the operator to torque the catheter past the region of difficulty. It should be noted that use of a $125-\mathrm{cm}$ catheter over a $180-\mathrm{cm}$ wire does cause the operator to lose access to the wire temporarily. This loss of access occurs because, when a $90-\mathrm{cm}$ guiding sheath is used with approximately 20 to $30-\mathrm{cm}$ of the wire advanced out of the sheath into the carotid artery, only 60 to $70-\mathrm{cm}$ of the $180-\mathrm{cm}$ wire is visible outside the patient. Thus, as the initial 60 to $70-\mathrm{cm}$ of the $125-\mathrm{cm} 5 \mathrm{~F}$ vertebral diagnostic (retrieval) catheter is being advanced, there is no exposed wire to stabilize. As such, it is critical to use fluoroscopy to watch for any unanticipated advancement of the AngioGuard device. This problem could be circumvented with use of a $300-\mathrm{cm}$ AngioGuard wire from the beginning of the procedure, but use of these longer wires is somewhat more cumbersome than that of the $180-\mathrm{cm}$ devices. This technique may also prove useful in the retrieval of other distal protection devices such as the Filter Wire EZ (Boston Scientific), the RX Accunet (Abbott Vascular, Abbott Park, Ill), and the Emboshield (Abbott Vascular). In conclusion, we believe that the use of a $5 \mathrm{~F}$ diagnostic catheter as a rescue device is an easy and readily available solution to failed retrieval of the AngioGuard distal protection device.

\section{References}

1. Beneficial effect of carotid endarterectomy in symptomatic patients with high-grade carotid stenosis. North American Symptomatic Carotid Endarterectomy Trial Collaborators. $N$ Engl J Med 1991;325:445-53

2. Endarterectomy for asymptomatic carotid artery stenosis. Executive Committee for the Asymptomatic Carotid Atherosclerosis Study. JAMA 1995;273:1421-28

3. Shawl FA. Emergency percutaneous carotid stenting during stroke. Lancet 1995;346:1223

4. Yadav JS, Wholey MH, Kuntz RE, et al. Protected carotid-artery stenting versus endarterectomy in high-risk patients. N Engl J Med 2004;351:1493-501

5. Cordis Corporation. Instructions for Use. AngioGuard XP Emboli Capture Guidewire System [package insert]. Miami Lakes, Fla: Cordis; 2006. Available at http:// www.cordislabeling.com/pdf/24408544_3.pdf. 